\title{
Lp-PLA 2 Selective Inhibitor (Darapladib) Effect In Lowering The Expression Level Of IL-IB And IL-6 In The Renal At Type 2 Diabetes Mellitus
}

This article was published in the following Dove Press journal: Vascular Health and Risk Management

\author{
Titin Andri Wihastuti (D) \\ Fitria Nugraha Aini (iD) 2,3 \\ Cholid Tri Tjahjono ${ }^{4}$ \\ Yuni Hendrati Sulfia ${ }^{3}$ \\ Zuhrotus Sholichah ${ }^{3}$ \\ Teuku Heriansyah (iD ${ }^{5}$ \\ 'Department of Basic Science in Nursing, \\ Faculty of Medicine, University of \\ Brawijaya, Malang, East Java, Indonesia; \\ ${ }^{2}$ Faculty of Medicine, University of Islam \\ Malang, Malang, East Java, Indonesia; \\ ${ }^{3}$ Master Programme of Biomedicine, \\ Faculty of Medicine, Brawijaya University, \\ Malang, East Java, Indonesia; ${ }^{4}$ Department \\ of Cardiology and Vascular Medicine, \\ Faculty of Medicine, Brawijaya University, \\ Malang, East Java, Indonesia; ${ }^{5}$ Department \\ of Cardiology and Vascular Medicine, \\ Faculty of Medicine, University of Syiah \\ Kuala, Banda Aceh, Aceh, Indonesia
}

Correspondence: Teuku Heriansyah Department of Cardiology and Vascular Medicine, Faculty of Medicine, Syiah Kuala University, St. Teuku Nyak Arief Darussalam, Banda Aceh, Aceh 23III, Indonesia

Tel +62 8I-|66I-8282

Email teuku_hery@unsyiah.ac.id
Purpose: The aim of this study is to prove that type 2 diabetes mellitus can induce increasing inflammation marker in renal and that the provision of darapladib as Lp-LA2 Inhibitor agents can inhibit inflammation that were measured from the expression of IL-1B and IL-6- type cytokine in renal. This study also discusses the correlation between IL-1B and IL-6- type cytokine expression in renal.

Methods: Thirty Sprague-Dawley (SD) rats were divided into three main groups; those are negative control group (NC), Type 2 Diabetes Mellitus group (T2DM) given high fat diet (HFD) with streptozotocin intraperitoneal injection $(35 \mathrm{mg} / \mathrm{kg} \mathrm{BW})$ and diabetes mellitus + darapladib group $(\mathrm{DM}+\mathrm{DP})$. Each group was treated within two serial treatment time: 8 weeks and 16 weeks. Expressions of $I L-1 B$ and $I L-6$ - type cytokine in renal were the markers that we measured by immunofluorosense method.

Results: The administration of darapladib can significantly decrease the expression of IL1B- type cytokine $(\mathrm{p}$ ANOVA $=0.029, \mathrm{p}<0.005)$ measured in rats' renal both at weeks 8 and 16 in the T2DM group. The Expression of IL-6- type cytokine also showed a significant difference after treated with darapladib both at weeks 8 and 16 in T2DM group with p-value of ANOVA $=0.033, \mathrm{p}<0.005$. The Pearson correlation showed a strong correlation (linear regression value was $\mathrm{r}^{2}=0.743$ ).

Conclusion: Our results show that atherosclerosis caused by inflammation in renal T2DM SD rats could be inhibited by the administration of darapladib.

Keywords: IL-1B- type cytokine, IL-6- type cytokine, kidney organ, diabetes mellitus type 2, darapladib

\section{Introduction}

Metabolic syndrome includes central obesity, insulin resistance, elevated blood pressure, impaired glucose tolerance, and dyslipidemia. ${ }^{1}$ Type 2 diabetes mellitus is dominated by insulin resistance followed by insulin deficiency or not. The cause of insulin resistance usually occurs in obesity. ${ }^{2}$ Type 2 diabetes mellitus is a metabolic disorder characterized by a combination of peripheral insulin resistance and insufficient insulin secretion by pancreatic beta cells. Insulin resistance is associated with elevated levels of free fatty acids and pro-inflammatory cytokines in plasma. Both will lead to a decrease in the transport of glucose into muscle cells and increased hepatic glucose production. ${ }^{3}$

Dyslipidemia is common in insulin resistance or type $2 \mathrm{DM}$, even with controlled blood sugar. Dyslipidemia is suspected to be associated with hyperinsulinemia. Increased lipolysis occurs in insulin resistance, resulting in increased free fatty acid in plasma 
which will further increase the free fatty acid uptake into the liver. ${ }^{4}$ The most important established risk factors for CKD are diabetes and hypertension. However, obesity and metabolic syndrome (MS) also affect independent predictors of CKD. ${ }^{5}$

The combination of hyperglycemia, hypertension, hyperlipidemia, and low-grade inflammation can cause metabolic derangements which may initiate changes in heart, pancreas, liver, and kidney. ${ }^{6}$ Previous research indicated that renal disease patients show increased circulating levels of non-specific markers of inflammation such as C-reactive protein (CRP) and pro-inflammatory cytokines such as IL-1 and IL-6. ${ }^{7}$ Inflammation is known as having a pivotal role in atherosclerosis development. ${ }^{8}$ The injury and inflammation are mediated by the release of macrophage-derived inflammatory cytokines like interleukin (IL)-1, IL-6, IL-23, and the generation of reactive oxygen/nitrogen species, each of which has been implicated in impaired renal function. ${ }^{9}$ IL-1B is known as an inflammatory marker that has an important role in the pathogenesis of atherosclerosis. ${ }^{10}$ Expression and secretion of IL-6 are regulated by IL-1 and TNF-a, which are highly induced in the atherosclerotic plaque. ${ }^{11}$

Interleukin-6 was first described as interferon $\beta 2$, hepatocyte stimulation factor, cytotoxic $\mathrm{T}$ cell differentiation factor, B-cell differentiation factor and B-2 cell stimulation factor, reflecting its capacity to regulate lymphocyte activation and acute phase response. ${ }^{12}$ Until now, IL-6 has family cytokines which have been identified: cardiotrophinlike cytokine (CLC), IL-6, oncostatin M (OSM), ciliary neurotrophic factor (CNTF), leukemia inhibitory factor (LIF), cardiotropin-1 (CT-1), neuropoietin (NP), IL-11, IL-27, and IL-31. ${ }^{13}$ Inflammatory induction of IL-6 was regulated by specific IL$1 \mathrm{~B}$ and IL- 6 gene variants that modulate atherosclerosis development and progression especially in renal disorders. IL-6 also had a critical role in pathogenesis of various forms of CKD suggested by both human and animal studies. ${ }^{14,15}$

IL-1 currently had 11 family members: IL- $1 \alpha$, IL-1 $\beta$, IL1Ra, IL-18, IL-33, IL-36Ra, IL-36 $\alpha$, IL-36 $\beta$, IL-36 $\gamma$, IL-37, and IL-38. The IL-1B cytokine also had other names, such as IL-1F2, leukocytic pyrogen, leukocytic endogenous mediator, mononuclear cell factor and lymphocyte activating factor. Its function is for Pro-inflammatory, Th17 cell response, and tissue repair. ${ }^{9}$ The major sources of IL- $1 \beta$ secretion are B lymphocytes, monocytes and macrophages, neutrophils, dendritic cells (DC), B lymphocytes, and natural killer (NK) and keratinocytes. ${ }^{16}$ Pro-atherogenic function of IL-1 ligands deficiency of IL-1R alleviated plaque formation in models of hyperlipidemia and induced bacteremia-accelerated atherosclerosis. This chronic inflammatory could induce organ impairment such as in kidney disease. ${ }^{17,18}$

The aim of this study is to prove that type 2 diabetes mellitus can induce increasing inflammation marker in renal and the provision of darapladib as Lp-LA2 Inhibitor agents can inhibit inflammation measured from the expression of IL-1B and IL-6- type cytokine in renal. This study also discusses the correlation IL-1B and IL-6- type cytokine expression in renal.

\section{Materials And Methods Study Design}

This study used Sprague-Dawley rats (4 weeks old, weighted around 150-200 g) obtained from Bogor Agricultural University, Indonesia. These rats were divided into three main groups: normal group (no high fat diet and no treatment of darapladib), high fat diet (HFD) group with streptozotocin intraperitoneal injection $(35 \mathrm{mg} / \mathrm{kg} \mathrm{BW})$ administered before blood glucose first measurement to induce T2DM and darapladib group (high fat diet and treatment with darapladib). Each group was treated within two serial treatment time: 8 and 16 weeks. Darapladib was purchased from GlaxoSmithKline. It was administered orally as much as $20 \mathrm{mg} / \mathrm{kg} /$ day according to time serial group. The control groups were given placebo. Normal rat food contained 3.43 $\mathrm{kcal} / \mathrm{g}$ total energy calories, while the high fat diet contained $5.29 \mathrm{kcal} / \mathrm{g}$ total calorie energy. Thirty grams of food were given to each rat every day. To ensure the type 2 diabetes mellitus (T2DM) model, we measured blood glucose, insulin resistance (HOMA-IR), and lipid profiles.

\section{Measurement Test To Ensure T2DM Model Blood Glucose Measurement}

We measured blood glucose level using GlucoDR blood glucose test meter (All Medicus Co., Ltd., Dongan-gu, Anyang-si, Korea). Fasting blood glucose levels were more than $7.5 \mathrm{mmol} / \mathrm{L}$ in rats or random blood glucose level of $10.4 \mathrm{mmol} / \mathrm{L}$ was confirmed as T2DM.

\section{Insulin Resistance Measurement}

Measuring insulin resistance can be performed using homeostatic model assessment-insulin resistance (HOMA-IR) formula especially in rats which required some data, such as fasting glucose and plasma insulin levels by the following formula. ${ }^{19}$

$$
\frac{F B S x F I N S}{14.1}
$$


HOMA-IR = Explanation: HOMA-IR: Homeostatic model assessment-insulin resistance FBG: Fasting blood glucose $(\mathrm{mmol} / \mathrm{L})$ FINS: Fasting insulin plasma $(\mu \mathrm{U} / \mathrm{L})$.

In the Interpretation of HOMA-IR calculation in rats, the result $>1.716$ can be categorized as insulin resistant with $83.87 \%$ sensitivity and $80.56 \%$ specificity (95\% confidence interval).

\section{Lipid Profil Measurement}

Measurement of LDL/VLDL, total cholesterol concentration, and HDL were performed using colorimetric method, EnzyChrom Assay Kit.

\section{Interleukin-IB (IL-IB) And Interleukin-6 (IL-6) Expression Measurement Using Immunofluorescence}

Il-1B and IL-6 expressions from renal tissue of rats were measured by immunofluorescence. The renal tissue was previously fixed with PHEMO buffer (68mM PIPES, $25 \mathrm{mM}$, HEPES, pH 6.9, 15 mMEGTA, 3 mM MgCl2, 10\% [v/v] dimethyl sulfoxide containing $3.7 \%$ formaldehyde, and $0.05 \%$ glutaraldehyde) and was processed by immunofluorescence labelling with anti-rat antibody IL-1B using rhodamin secondary antibody (BIOS Inc. Boston, MA, USA) and antirat antibody IL-6 using FITC secondary antibody (Santacruz, USA). This parameter was observed with confocal laser scanning microscopy (Olympus Corp., Tokyo, Japan) and was quantitatively analyzed using Olympus FluoView Software (version 1.7A; Olympus Corporation) which generated numeric data in international unit, Arbitrary Unit (AU).

\section{Ethical Clearance}

All experiments were conducted according to the principle expressed in the 'Guiding Principles on the Care and Use of Animals'. The study received prior approval from the ethics committee of the Animal Care and Use Committee Brawijaya University, evaluated and approved by Indonesian Health Research Ethics Committee through approval No. 267/EC/KEPK-S1-PD/07/2017.

\section{Statistical Analysis}

IL-1B and IL-6 expression were obtained, and normality test was performed to determine the normality of the data. The data of expressions were analyzed using one-way ANOVA to determine the effect of darapladib administration and the interaction between times in Sprague-Dawley rats renal. This analysis was followed by Post Hoc using LSD method to detect parameter differences in each treatment group. Statistical analysis was performed using SPSS software version 21 (IBM Corporation, USA).

\section{Results}

We measured fasting blood glucose, insulin resistance, and lipid profile to ensure that the rats were developing T2DM. The result can be seen in our previous publication by Heriansyah (2017). T2DM model was made successfully if fasting blood glucose $>126 \mathrm{mg} / \mathrm{dl}$ and insulin resistance (using HOMA-IR) results $>1.716 .^{11} 20 \mathrm{mg} / \mathrm{kg} \mathrm{BW}$ /day of darapladib given to T2DM group showed a decreasing in LDL and total cholesterol and an increase in HDL both in 8 weeks and 16 weeks. Level of blood glucose also showed a decrease in 8 weeks and 16 weeks of darapladib administration. From these results, we also concluded that Sprague-Dawley T2DM model was successfully made.

The T2DM group treated with $20 \mathrm{mg} / \mathrm{kg} \mathrm{BW} /$ day of darapladib had a decreasing expression of IL-1B compared to the T2DM group in 8 weeks (Figure 1). ANOVA test with $95 \%$ confidence interval showed that there were significant differences in the expressions of IL-1B between T2DM and DMDP group in 8 weeks with $p<0.05$. The result in 16 weeks (Figure 1) also showed the significant differences in expressions of IL-1B in T2DM compared to DM treated with darapladib group (with $\mathrm{p}<0.05$ ).

The result of IL-6 expressions also showed that DMDP group has lower expression than T2DM group in 8 weeks with significant value $(\mathrm{p}<0.05)$ (Figure 2$)$. These results as well as the group in 16 weeks which had a significant difference between T2DM group compared to DM treated with darapladib. The results of Pearson correlation analysis showed that there was a strong correlation between IL-1B and IL-6 expressions in renal tissue with $\mathrm{R}^{2}=0.743, \mathrm{P}<0.05$ (Figure 3).

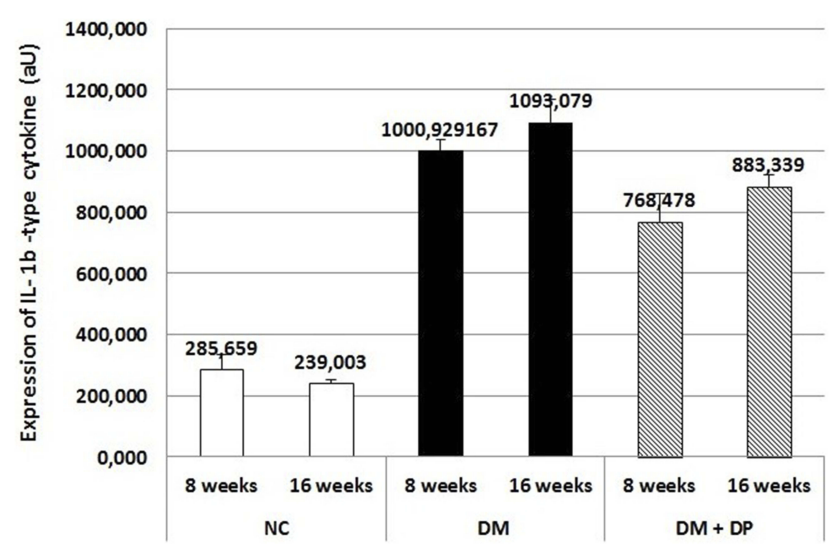

Figure I The Expression of IL-IB- type cytokine of 8 and 16 weeks serial treatment time on kidney (P ANOVA $=0.029)$. 




Figure 2 The Expression of IL-6- type cytokine of 8 and 16 weeks serial treatment time on kidney (P ANOVA $=0.033$ ).

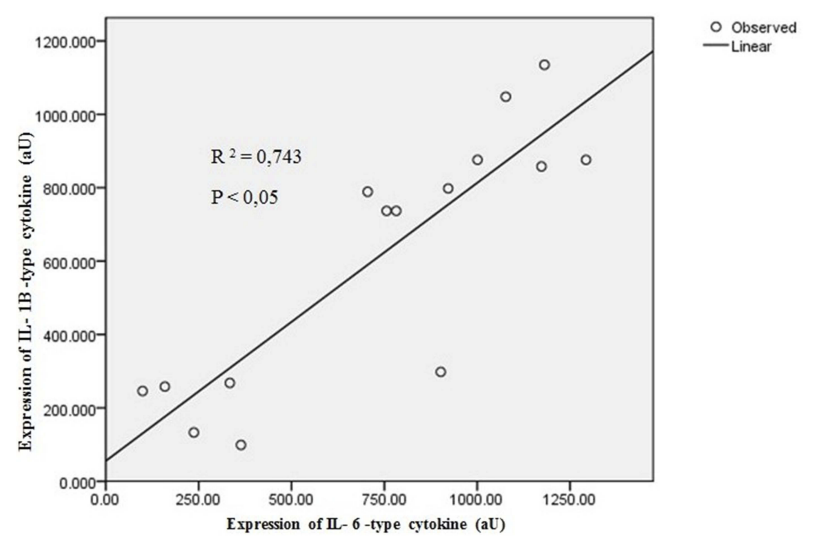

Figure 3 Linear regression of kidney IL-IB and IL-6 type cytokine.

Abbreviations: IL-IB, interleukin I beta; IL-IB, interleukin 6; aU, arbitrary unit; NC, negative control; DM, diabetes mellitus; DP, darapladib.

\section{Discussion}

Metabolic syndrome includes central obesity, insulin resistance, elevated blood pressure, impaired glucose tolerance, and dyslipidemia. ${ }^{1}$ These conditions, combined with lowgrade inflammation, caused metabolic derangements which may initiate changes in heart, pancreas, liver, and kidney. ${ }^{6,7}$ Diabetes mellitus is well known as a risk factor for the development of acute renal damage. ${ }^{20}$ Peripheral insulin resistance and/or impaired insulin secretion are causative factors for diminished carbohydrate metabolism. Glucose intolerance is commonly found in patients and animals affected by renal impairment. ${ }^{21}$ Previous research stated that renal disease patients show an increased circulating level of non-specific markers of inflammation such as C-reactive protein (CRP) and pro-inflammatory cytokines such as IL-1 and IL-6. ${ }^{9,12}$

Atherosclerosis plaque formation in renal is regulated by inflammation in T2DM. ${ }^{22}$ The state of Hyperglycemia can increase NADPH oxidase complex which results in increased production of reactive oxygen species (ROS) and triggers OxLDL resulting in increased oxidative stress and release NF- $\mathrm{KB}$ from the cytoplasm to the nucleus that causes the transactivation of multiple pro-inflammatory agents. ${ }^{23-25}$ Lyso PC and ox NEFA produced by Lp-PLA2 action through ox-LDL are able to promote monocyte migration by inducing monocyte chemotactic protein (MCP)-1 and stimulating Interleukin (IL)-1 $\beta$, IL-6, and tumor necrosis factor (TNF)- $\alpha$ production and scavenger receptor expression in macrophages in a concentrationdependent manner. ${ }^{11}$ Hyperglycemia also increases the NF- $\mathrm{kB}$ level, resulting in increased levels of CAMs. NF- $\mathrm{kB}$ regulates the increase of other inflammatory markers like TNF- $\alpha$, MCP1, IL-1, and IL-6. ${ }^{25-27}$ The injury and inflammation are mediated by the release of macrophage-derived inflammatory cytokines like interleukin (IL)-1, IL-6, IL-23, and the generation of reactive oxygen/nitrogen species; each of which have been implicated in impaired renal function. IL-1B is known as an inflammatory marker that has an important role in the pathogenesis of atherosclerosis. ${ }^{28,29}$ Interleukin-6 (IL-6) associated with endothelial damage can initiate atherosclerotic events. Expression and secretion of IL-6 are regulated by IL1 and TNF-a, which are highly induced in the atherosclerotic plaque. ${ }^{9}$ Inflammatory induction of IL- 6 was regulated by specific IL-1B and IL-6 gene variants that modulate atherosclerosis development and progression, especially in renal disorders. ${ }^{22}$

This study confirmed that darapladib as an inhibitory agent of Lp-PLA2 can decrease the inflammation process in renal T2DM rats. The results showed that IL-1B and IL-6 expressions decrease significantly in T2DM treated with darapladib in 8 weeks and 16 weeks. These results are consistent with in vivo studies by Wang et al (2011) which concluded that oral darapladib induced a significant decrease of IL-6. Darapladib reduced the expression of Lp-PLA2 to interact with the catalytic residues of Lp-PLA2. ${ }^{30}$ Atherosclerotic lesion treated with darapladib was able to demonstrate the improvement of the lesions associated with decreased activity of Lp-PLA2. ${ }^{27}$ This therapy holds promise in cardiovascular disease. Atherosclerosis is known to have a chronic process which requires a long-term treatment. ${ }^{31}$

Pearson correlation test showed that IL-1B and IL-6 had $\mathrm{r}^{2}=0.743$. That value defined that IL-1B and IL-6 had a strong correlation. The increase of IL-1B type cytokine expression will also increase IL-6-type cytokine expression. This result is similar to that of other research which showed that both cytokines were pro-inflammatory cytokines. Further, the increase of both IL-1B and IL-6 will increase the inflammation process 
in the kidney. Then, it would increase the risk factor of people with T2DM and Dyslipidemia who had high risks of kidney disease and other complication.

Treatment with darapladib has been evaluated in diabetes and hypercholesterolemia (DM-HC) models in pigs. It resulted in a considerable decrease in plaque area and a markedly reduced necrotic core area. The Phase II Clinical trial research has also shown that the inhibition of lp-PLA2 with darapladib has arrested the expansion of the necrotic core, a key determinant of plaque vulnerability, despite not preventing plaque formation (Miao-miao et al, 2011). Another Phase II study also indicated that IL- 6 could be reduced by darapladib administration. Meanwhile, there were disappointing results of Lp-PLA 2 inhibitor (darapladib) which failed to reduce the risk of secondary major coronary events when added to optimal medical therapy in both SOLID-TIMI 52 and STABILITY studies. In both SATBILLITY and SOLID TIMI 52 studies, patients were treated with darapladib orally for secondary prevention of CHD and monitored every 6 months. There was only a small benefit in the secondary end points - a $10 \%$ reduction in the risk of major coronary events and a $9 \%$ reduction in total coronary events - in the STABILITY study. However, the findings were considered exploratory, and of uncertain significance in the absence of effect on the primary endpoint. ${ }^{32}$

This is the difference between this study and the Phase III STABILITY study. The Phase III trial, SOLID-TIMI 52 over 13.000 patients received darapladib within a month of heart attack and evaluated for cardiovascular death, reduced in heart and stroke attack. However, none of the studies demonstrated the benefit. ${ }^{33-35}$ In this study, we gave the darapladib to DMT2 rats model which have a high risk to develop macrovascular complication such as atherosclerosis or microvascular complication like a diabetic nephropathy. It showed that darapladib can prevent atherosclerosis in DMT2 rats by reducing renal IL-6 and IL-1B as inflammation markers. Our study suggests that Darapladib as an anti-inflammation therapy can be used for the prevention of cardiorenovascular disease and lp-PLA2 is a promising target against atherosclerosis. This can be the reason of the darapladib clinical trial failure. Darapladib did not have effective actions in late or chronic atherosclerosis. On the contrary, it had good effects in the early phase of atherosclerosis.

\section{Conclusion}

From this study, we are able to predict integrated data for IL-6 and IL-1B tissue expression in a risk factor of atherosclerosis using T2DM rat models. Our results show that atherosclerosis caused by inflammation in renal T2DM SD rats could be inhibited by the administration of darapladib in diabetes mellitus condition. In summary, darapladib attenuated the inflammatory burden, resulting in the prevention of atherosclerosis in T2DM Sprague-Dawley rats.

\section{Acknowledgment}

This study is supported by the Ministry of Research, Technology, and Higher Education of Indonesia.

\section{Disclosure}

The authors report no conflicts of interest in this work.

\section{References}

1. Suman RK, Mohanty IR, Borde MK, Maheshwari U, Deshmukh YA. Development of an experimental model of diabetes co-existing with metabolic syndrome in rats. Adv Pharmacol Sci. 2016;2016:9463476.

2. Zhang X, Lerman LO. The metabolic syndrome and chronic kidney disease. Transl Res Elsevier Inc. 2017;183:14-25. doi:10.1016/j. trs1.2016.12.004

3. American Diabetes Association. Diagnosis and classification of diabetes mellitus. Diabetes Care. 2013;36(SUPPL.1):67-74. doi:10.2337/dc13S067

4. Arca M, Pigna G, Favoccia C. Mechanisms of diabetic dyslipidemia: relevance for atherogenesis. Curr Vasc Pharmacol. 2012;10(6):684 686. doi:10.2174/157016112803520864

5. Yoon YS, Park HS, Yun KE, Kim SB. Obesity and metabolic syndrome-related chronic kidney disease in nondiabetic, nonhypertensive adults. Metabolism. 2009;58(12):1737-1742. doi:10.1016/j. metabol.2009.05.029

6. Zhang S, Ritter LR, Ibragimov AI. Foam cell formation in atherosclerosis: HDL and macrophage reverse cholesterol transport. Discret Contin Dyn Syst - Ser S. 2013;2013:825-835.

7. Kuate D, Kengne APN, Biapa CPN, Azantsa BGK, Abdul Manan Bin Wan Muda W. Tetrapleura tetraptera spice attenuates high-carbohydrate, high-fat diet-induced obese and type 2 diabetic rats with metabolic syndrome features. Lipids Health Dis.2015;14:50. BioMed Central. doi:10.1186/s12944-015-0051-0

8. Wihastuti TA, Sargowo D, Widodo MA, Heriansyah T, Soeharto S, Ardi AI. Subchronic toxicity of ganoderma lucidum polysaccharide peptide (PsP) to liver physiology and histopathology imaging of liver on rattus norvegicus strain wistar rats. Biomed Pharmacol J. 2014;7 (2):417-424. doi: $10.13005 / \mathrm{bpj} / 506$

9. Pfeilera S, Winkelsb H, Kelma M, Norbert G. IL-1 family cytokines in cardiovascular disease. Cytokine. 2017; In press:22.

10. Heriansyah T, Siswanto BB, Santoso A, et al. Ox-LDL, lysophospatidyl choline platelet activating factor, il-6 expression level and foam cell number with darapladib treatment in early development of atherosclerosis: in vivo study for type 2 diabetes mellitus model. $J$ Hong Kong Coll Cardiol. 2016;24(2):64-73.

11. Heriansyah T, Wihastuti TA, Siswanto BB, et al. Lowering inflammation level by Lp-PLA2 inhibitor (Darapladib) in early atherosclerosis development : in vivo rat type 2 diabetes mellitus model. J Cardiovasc Dis Res. 2017;8(2):50-55. doi:10.5530/jcdr.2017.2.12

12. Feigerlová E, Battaglia-Hsu S-F. IL-6 signaling in diabetic nephropathy: from pathophysiology to therapeutic perspectives. Cytokine Growth Factor Rev. 2017;37:57-65. doi:10.1016/j.cytogfr.2017.03.003

13. Su H, Lei C-T, Zhang C. Interleukin-6 signaling pathway and its role in kidney disease: an update. Front Immunol. 2017;8(April):1-10. doi:10.3389/fimmu.2017.00001 
14. Mack M. Inflammation and fibrosis. Hepatol Int Soc Matrix Biol. 2013;58(S1):281A- 284A.

15. Zhang W, Wang $\mathrm{W}, \mathrm{Yu} \mathrm{H}$, et al. Interleukin 6 underlies angiotensin II-induced hypertension and chronic renal damage. Hypertension. 2012;59(1):136-144. doi:10.1161/HYPERTENSIONAHA.111.173 328

16. Garley M, Jabłońska E, Sawicka-Powierza J, et al. Expression of IL-1 and IL-6 and their natural regulators in leukocytes of B-cell chronic lymphocytic leukaemia patients. Adv Med Sci. 2016;61(2):187-192. doi:10.1016/j.advms.2015.12.006

17. Wolf G. Molecular mechanisms of angiotensin II in the kidney: emerging role in the progression of renal disease: beyond haemodynamics. Nephrol Dial Transplant. 2013;13(5):1131-1142. doi:10.1093/ndt/13.5.1131

18. Kochi M, Kohagura K, Shiohira Y, Iseki K, Ohya Y. Chronic kidney disease, inflammation, and cardiovascular disease risk in rheumatoid arthritis. J Cardiol. 2017. doi:10.1016/j.jjcc.2017.08.008

19. Cacho J, Sevillano J, de-Castro J, Herrera E, Ramos M. Validation of simple indexes to assess insulin sensitivity during pregnancy in wistar and Sprague-Dawley rats. Am J Physiol Endocrinol Metab. 2008;295:1269-1276. doi:10.1152/ajpendo.90207.2008

20. Ozbilgin S, Ozkardesler S, Akan M, et al. Renal ischemia/reperfusion injury in diabetic rats : the role of local ischemic preconditioning. Biomed Res Int. 2016;2016:8580475. doi:10.1155/2016/8580475

21. Leblond A, Lesage S, Cartee GD, Pichette V. Mechanism of insulin resistance in a rat model of kidney disease and the risk of developing type 2 diabetes. PLoS One. 2017;12:1-17.

22. Hussein M, Mahmoud G, Ibrahim G, Aly D, Mohammad A. Possible association of interleukin-1beta $(-511 \mathrm{C} / \mathrm{T})$ and interleukin-6 $(-174 \mathrm{G} /$ C) gene polymorphisms with atherosclerosis in end stage renal disease Egyptian patients on maintenance haemodialysis. Egypt J Med Hum Genet. 2013;14(3):267-275. doi:10.1016/j.ejmhg.2013.04.001

23. Wihastuti TA, Sargowo D, Heriansyah T, Aziza YE, Iwana AN, Evitasari LA. The reduction of aorta histopathological images through inhibition of reactive oxygen species formation in hypercholesterolemia rattus norvegicus treated with polysaccharide peptide of Ganoderma lucidum. Iran J Basic Med Sci. 2014;18(9):514-519.

24. Lawrence T. The nuclear factor NF-kappaB pathway in inflammation. Cold Spring Harb Perspect Biol. 2009;1(6):a001651. doi:10.1101/ cshperspect.a001651
25. Heriansyah T, Hanifa H, Andarini S, Wihastuti TA. Darapladib inhibit adhesion molecule expression in aorta at early stages of atherosclerosis using Sprague-Dawley type 2 diabetes mellitus model. Asian J Pharma Clin Res. 2017;10(6):4-7.

26. Heriansyah T, Adam AA, Wihastuti TA, Rohman MS. Elaborate evaluation of serum and tissue oxidized LDL level with darapladib therapy: a feasible diagnostic marker for early atherogenesis. Asian Pac J Trop Biomed. 2017;7(2):134-138. doi:10.1016/j.apjtb.2016.11.014

27. Heriansyah T, Wihastuti TA, Anita K, et al. Atherogenesis inhibition by darapladib administration in dyslipidemia model Sprague-Dawley rats. Natl J Physiol Pharm Pharmacol. 2015;6(1):1-7.

28. Wang WY, Zhang J, Wu WY, et al. Inhibition of lipoprotein-associated phospholipase a2 ameliorates inflammation and decreases atherosclerotic plaque formation in apoE-deficient mice. PLoS One. 2011;6:8.

29. Pashkow FJ. Oxidative stress and inflammation in heart disease:do antioxidants have a role in treatment and/or prevention? Int J Inflam. 2011;2011(Figure 4):514623.

30. Wihastuti TA. Vasa vasorum anti-angiogenesis through $\mathrm{H} 2 \mathrm{O} 2, \mathrm{HIF}-1 \alpha$, $\mathrm{NF}-\kappa \mathrm{B}$, and iNOS inhibition by mangosteen pericarp ethanolic extract (Garcinia mangostana Linn) in hypercholesterol- diet-given Rattus norvegicus Wistar strain. Vasc Health Risk Manag. 2014;10:523-531.

31. Ridker PM. Targeting inflammatory pathways for the treatment of cardiovascular disease. Eur Heart J. 2014;35:540-543.

32. Hassan M. STABILITY and SOLID-TIMI 52: lipoprotein associated phospholipase A 2 (Lp-PLA 2) as a biomarker or risk factor for cardiovascular diseases. Global Cardiol Sci Pract. 2015;6. doi: $10.5339 / \mathrm{gcsp} .2015 .6$

33. Mohler ER, Ballantyne CM, Davidson MH, et al. The effect of darapladib on plasma lipoprotein-associated phospholipase A2 activity and cardiovascular biomarkers in patients with stable coronary heart disease or coronary heart disease risk equivalent. the results of a multicenter, randomized, double-Bl. J Am Coll Cardiol. 2008;51 (17):1632-1641. doi:10.1016/j.jacc.2007.11.079.

34. O’Donoghue ML, Braunwald E, White HD, et al. Effect of darapladib on major coronary events after an acute coronary syndrome: the SOLID-TIMI 52 randomized clinical trial. JAMA. 2014;312 (10):1006-1015. doi:10.1001/jama.2014.11061

35. White HD, Held C, Stewart R, et al. Darapladib for preventing ischemic events in stable coronary heart disease. NEngl $\mathrm{J}$ Med. 2014;370(18):page 1702-1711.
Vascular Health and Risk Management

\section{Publish your work in this journal}

Vascular Health and Risk Management is an international, peerreviewed journal of therapeutics and risk management, focusing on concise rapid reporting of clinical studies on the processes involved in the maintenance of vascular health; the monitoring, prevention and treatment of vascular disease and its sequelae; and the involvement of metabolic disorders, particularly diabetes. This journal is indexed on PubMed Central and MedLine. The manuscript management system is completely online and includes a very quick and fair peerreview system, which is all easy to use. Visit http://www.dovepress. com/testimonials.php to read real quotes from published authors. 